\title{
Systematics and bioacoustics of the Poecilimon sanctipauli-group (Orthoptera: Tettigonioidea: Phaneropteridae)
}

\author{
Klaus-Gerhard HELLER ${ }^{1}$ and Hasan SEVGILI ${ }^{2}$ \\ ${ }^{1}$ Grillenstieg 18, 39120 Magdeburg, Germany; e-mail: heller.volleth@t-online.de \\ ${ }^{2}$ Harran Üniversitesi, Fen-Edebiyat Fakültesi, Biyoloji Bölümü, Osmanbey Kampüsü, Sanliurfa, Turkey; \\ e-mail: hsevgili@harran.edu.tr
}

Key words. Poecilimon, morphology, bioacoustics, Tettigonioidea, bush-crickets, Turkey, Greece

\begin{abstract}
In this paper a combination of characters by which Poecilimon species (Orthoptera: Tettigonioidea: Phaneropteridae) can be recognised as members of the $P$. sanctipauli group are described. Most important are the wide fastigium, short ovipositor and song characters. The morphological characters are figured and described (Table 1), and the song patterns illustrated by oscillograms. The proposed phylogenetic relationships of the members of this group are written as $[P$. mytilenensis $(P$. pulcher, $P$. lodosi, $P$. sanctipauli)]. All species of the group are known from southwest Turkey and some east Aegean islands. The three species $P$. pulcher, $P$. lodosi and $P$. sanctipauli are morphologically and bioacoustically quite similar. $P$. sanctipauli and $P$. pulcher are distinct species, $P$. lodosi, however, possesses a combination of the key characters of the other two species. It may be a relict species or, in our opinion more probably, a species of hybrid origin.
\end{abstract}

\section{INTRODUCTION}

The genus Poecilimon is one of the most species-rich genera of Orthoptera in the Palearctic. Like the other members of the subfamily Barbitistinae all species are micropterous and unable to fly. This fact is certainly in part responsible for the high number of species. The phylogenetic relationships within this huge genus (about 120 species), however, are not well understood. Ramme (1933) published the first revision of Poecilimon, establishing 8 groups for the 68 species known at that time. However, he made no attempt to clarify the relationships between the groups and between the species within the groups. Bey-Bienko (1954) recognized 73 species in this genus and presented a key and detailed re-descriptions of especially the Russian and Anatolian species. Because he stated that Ramme's groups were largely artificial, he abandoned the attempt to divide the genus into groups. Thirty years later, Heller (1984a) presented a cladogram of the relationships of 35 European forms using apomorphic characters. For a better understanding of the evolution of the genus, however, knowledge of the phylogenetic position of more of the species is necessary. In addition to conventional morphological characters it is now appreciated that bioacoustic data can help clarify the relationships of species and subspecies. Besides characters of the song pattern the structures used for sound production (male stridulatory file, size of female tegmina) and for hearing (size of prothoracic auditory spiracles) are also important for the identification and grouping of species (Heller, 1984a, 1988; Willemse \& Heller, 1992; Heller \& Lehmann, 2004).

Because of the large number of species involved, we concentrate on identifying and studying small monophyletic groups and clarifying the relationships of the species within these groups (e.g. Lehmann, 1998; Heller \& Lehmann, 2004).

In the present paper we focus on a small group of species near P. sanctipauli Brunner von Wattenwyl, 1878. Ramme (1933) placed this species in a subgroup of his group $\mathrm{V}$ together with $P$. pulcher and $P$. mytilenensis. $P$. lodosi, described by Harz (1975), also belongs to this group (see below). All species of this group are known from southwest Turkey and some east Aegean islands.

\section{MATERIAL AND METHODS}

We have studied specimens preserved in the following collections: AZME - Ankara Zirai Mücadele Enstitüsü, Ankara, Turkey; $\mathrm{CH}$ (followed by the specimen code) - Collectio Heller; HUZOM - Hacettepe Üniversitesi Zooloji Müzesi, Ankara, Turkey; MIZT - Italy, Museo ed Istituto di Zoologioca sistematica della Universitata di Torino. Other material is deposited in MZAA - Michigan University Museum of Zoology, Ann Arbor, USA; MHNG -Muséum d'Histoire Naturelle, Genève, Switzerland; NHMW - Naturhistorisches Museum, Vienna, Austria, ZMHB - Museum für Naturkunde der Humbolt-Universität, Berlin, Germany; ZMUH - Zoologisches Museum Universität Hamburg, Hamburg, Germany.

At DORSA the localities (except some from Turkey) of all specimens in $\mathrm{CH}$ can be visualised by a web-based GIS mapping tool (including lat/long data). At SysTax the digitised sound recordings of many specimens are available.

Morphological structures were examined and measured using an Olympus stereomicroscope. For sound recording in the field an Uher tape recorder 4200 IC with a Uher M645 microphone was used (frequency response flat up to $20 \mathrm{kHz}$ ), in the laboratory a Racal store 4 D tape recorder with Brüel \& Kjaer 4133 and 4135 microphones (frequency response flat up to 40 resp. $70 \mathrm{kHz}$ ). Oscillograms and sound analysis were made using a $\mathrm{PC}$ and the programs Turbolab (Stemmer AG) and CoolEdit. Wing movements were recorded by an opto-electronic device (Heller, 1988). The stridulatory files were studied using scan- 
ning electron microscopes Autoscan, Fa. ETEC SEM, and DSM 960, Fa. Zeiss.

Bioacoustic terminology: Calling song: spontaneous song produced by an isolated male. Syllable: the song produced by one opening-closing movement cycle of the tegmina. Impulse: a simple, undivided, transient train of sound waves generated by the impact of one tooth of the stridulatory file.

\section{RESULTS}

\section{Characteristics of the Poecilimon sanctipauli-group}

From a direct comparison of most European and Anatolian Poecilimon species (e.g., Heller, 1984a, 1988; Willemse \& Heller, 1992; Heller \& Reinhold, 1992; Lehmann, 1998; Sevgili, 2001; Heller \& Lehmann, 2004; Heller et al., in press) and the literature, it became evident that a group of species of this genus can be separated and recognised by the following characters:

1. Fastigium wider or as wide as scapus.

2. Relatively short ovipositor (ratio hindfemur to ovipositor above 2).

3. Relatively large to medium body size compared to other Poecilimon species.

4. Large prothoracic spiracles.

5. Tegmina of female overlapping, thus females able to respond acoustically.

6. Two-phase stridulatory movement: slow beginning, fast ending.

7. Song consisting of long syllables (ca. 0.1-2 s) containing many (ca. 35-200) impulses.

Members of the Poecilimon sanctipauli-group are recognised by a combination of characters [especially (1) and (2)]. None of these characteristics can be considered as a unique autapomorphic character, some are probably even plesiomorphic characters. The evolution of the wide fastigium (1) is difficult to evaluate. The same variation in fastigium width is also found in the closely related genus Isophya. In Poecilimon, however, a wide fastigium is found only in the P. sanctipauli and P. ornatus/affinis groups. In addition, there are two small Poecilimon species in Turkey, $P$. glandifer Karabag, 1950 and $P$. ataturki Ünal, 1999, with wide fastigia (see Ünal, 2003), but they belong to a group with mute females, possibly the $P$. ampliatus group (Heller \& Lehmann, 2004). The $P$. sanctipauli and $P$. ornatus/affinis groups show many similarities, but also distinct differences. One important difference is the length of the ovipositor (2). The ratio hindfemur to ovipositor is always below 2 in the $P$. ornatus/affinis group and above 2 in the $P$. sanctipauli group (data for the $P$. ornatus/affinis group used mean values from Harz, 1969). A short ovipositor, however, is also found in other groups of Poecilimon, and its evolution is again difficult to evaluate. It may be an adaptation to climate and change quite fast (see Heller et al., 2004). Body size (3) is another character difficult to assign as either an apomorphic or plesiomorphic state. The characters (4)-(5) are connected with communication and are plesiomorphic characters for the $P$. sanctipauli group and present in a relatively large number of Poecilimon species. Stumpner \& Heller (1992) give a description of the differences in spiracle size in Poecilimon and of the consequences for hearing. A two-phase stridulatory movement (6) was assumed to be an apomorphic character for a large group of Poecilimon species by Heller (1984a). This group includes the $P$. ornatus/affinis and $P$. sanctipauli groups. Most members of both groups share a calling song consisting of syllables with many impulses (7). It may be that the P. sanctipauli group is part of the $P$. ornatus/affinis group occurring in the south-east. However, a preliminary DNA study of Poecilimon lodosi, a member of the $P$. sanctipauli group, indicates it falls outside the very widely defined $P$. ornatus/affinis group. In any case, the species with the above-mentioned combination of characters belong together.

Distribution. Southwest Anatolia and some Aegean islands (see map: Fig. 10).

\section{Species of the Poecilimon sanctipauli-group}

1. Poecilimon sanctipauli Brunner von Wattenwyl, 1878

2. Poecilimon pulcher Brunner von Wattenwyl, 1891

3. Poecilimon lodosi Harz, 1975

4. Poecilimon mytilenensis Werner, 1932

a. P. mytilenensis mytilenensis Werner, 1932

b. P. mytilenensis brevissimus Heller, Willemse \& Sevgili, 2005

\section{Poecilimon sanctipauli Brunner von Wattenwyl, 1878}

Poecilimon sanctipauli Brunner von Wattenwyl, 1878: 37.

Type locality. Turkey: Izmir, Efes ("Ephesus").

Type depository. NHMW, syntypes $\left(60^{\star}, 7 q\right.$; not seen; contrary to the opinion of Otte (1997) and OSF2 no syntype was selected as lectotype).

\section{= Isophya isidori Giglio-Tos, 1914: 4.}

Type locality. Greece: Rhodos, Ag. Isidhoros.

Type depository. MIZT, holotype ơ (examined in 1984).

P. sanctipauli: Brunner von Wattenwyl, 1878: 37 (key), 40-41 (description); Brunner von Wattenwyl, 1891: 27 (key); Jacobson, 1905: 332 (key), 362 (description; in Russian); Kirby, 1906: 380 (catalogue); Werner, 1901: 286 (distribution); GiglioTos, 1914: 3 (distribution); Werner, 1933: 190 (distribution); Ramme, 1933: 507, 509 (arrangement), 510 (checklist), 535-6 (description); Werner, 1936: 10-11 (distribution); Jannone, 1936: 145 (distribution); Chopard, 1939: (distribution; not seen); Bey-Bienko, 1954: 256 (key), 288-9 (description); Karabag, 1958: 25 (faunistic catalogue); Harz, 1969: 102, 108 (key), 144 (description); Karabag et al., 1971: 77 (distribution); Karabag et al., 1981: 5 (distribution); Willemse, 1982: 156 (checklist), 188 (reference); 1984: 15 (checklist), 41 (reference); 1985: 41 (key), 266 (checklist); Heller, 1984b: 75 (distribution); Heller \& Helversen, 1986: 190-194 (acoustic communication); Heller, 1988: 45 (song); Willemse \& Heller, 1992: 301 (checklist); Tazegül \& Önder, 1995: 3 (distribution); Otte, 1997: 125 (catalogue); Heller et al., 1998: 30 (checklist); Ünal, 1999: 244 (distribution); Önder et al., 1999: 247 (distribution); Naskrecki \& Otte, 1999: (catalogue); Çıplak et al., 1999: 752 (checklist, distribution); Çıplak et al., 2002: 685 (checklist, distribution).

Databanks: DORSA, SysTax, OSF2.

Isophya isidori: Giglio-Tos 1914: 4 (description); Ramme, 1951: 137 (not to Isophya); Harz, 1969: 740 (species incertae sedis); Willemse, 1984: 30 (reference); Heller, 1984b: 75 (syn. of $P$. sanctipauli); Willemse 1985: 10 (as syn. of $P$. sanctipauli); Heller 1988: 45 (as syn of $P$. sanctipauli); Otte 1997: 126 (as syn of P. sanctipauli; catalogue); Naskrecki \& Otte 1999: (as syn of P. sanctipauli; catalogue) 
Diagnosis/description. See Table 1-2 and Figs 1-4.

Song. The calling song of the male consists of isolated syllables produced at intervals of several seconds. Each syllable consists of two separate impulse groups. The first long and crescending syllable results from the slow closing movement of the tegmina at the beginning, the second much shorter one from the fast final closure at the end of the movement cycle (Fig. 8; see also Heller \& Helversen, 1986; Heller, 1988). From the knowledge of the stridulatory movement and the structure of the stridulatory file it is possible to predict the process of sound production. During the first part of the syllable the

TABLE 1. Morphological differences of the species of the Poecilimon sanctipauli group.

\begin{tabular}{|c|c|c|c|c|}
\hline Species/Characters & P. sanctipauli & P. pulcher & P. lodosi & P. mytilenensis \\
\hline Body length $(\mathrm{mm})$ & $20-32$ & $22-33$ & $27-33$ & $16-24$ \\
\hline \multirow[t]{2}{*}{ Fastigium of vertex } & \multicolumn{3}{|c|}{ As wide as scapus or slightly wider, with dorsal groove } & \multirow{2}{*}{$\begin{array}{l}\text { As wide as scapus, with } \\
\text { lateral margins parallel or } \\
\text { slightly converging anteri- } \\
\text { orly, smooth above }\end{array}$} \\
\hline & $\begin{array}{l}\text { usually divergent } \\
\text { anteriorly }\end{array}$ & divergent anteriorly & $\begin{array}{l}\text { divergent anteriorly or } \\
\text { with subparallel lateral } \\
\text { sides }\end{array}$ & \\
\hline \begin{tabular}{|l|} 
Pronotum \\
(from above)
\end{tabular} & $\begin{array}{l}\text { Prozona slightly widening } \\
\text { anteriorly }\end{array}$ & $\begin{array}{l}\text { Prozona with lateral mar- } \\
\text { gins parallel or sometimes } \\
\text { slightly widening anteri- } \\
\text { orly } \\
\text { than prozona, constricted in } 1\end{array}$ & $\begin{array}{l}\text { Prozona slightly widening } \\
\text { anteriorly }\end{array}$ & $\begin{array}{l}\text { Pronotum constricted in } \\
\text { mesozonal region, meta- } \\
\text { zona not wider than pro- } \\
\text { zona }\end{array}$ \\
\hline $\begin{array}{l}\text { Pronotum } \\
\text { (in profile) }\end{array}$ & $\begin{array}{l}\text { Dorsal surface of pro- } \\
\text { notum distinctly concave, } \\
\text { metazona more raised than } \\
\text { prozona }\end{array}$ & $\begin{array}{l}\text { Dorsal surface of pro- } \\
\text { notum relatively straight } \\
\text { from fore margin to trans- } \\
\text { verse sulcus then slightly } \\
\text { raised in metazonal region }\end{array}$ & $\begin{array}{l}\text { Dorsal surface of pro- } \\
\text { notum slightly concave, } \\
\text { metazona more raised than } \\
\text { prozona }\end{array}$ & $\begin{array}{l}\text { Dorsal surface of pronotum } \\
\text { relatively straight from } \\
\text { fore margin to transverse } \\
\text { sulcus then slightly raised } \\
\text { in metazonal region }\end{array}$ \\
\hline \multirow[t]{2}{*}{ Paranota } & \multicolumn{3}{|c|}{ nearly 3 times as long as high } & \multirow{2}{*}{$\begin{array}{l}\text { nearly } 2.3 \text { times as long as } \\
\text { high; hind margin of para- } \\
\text { nota slightly convex }\end{array}$} \\
\hline & $\begin{array}{l}\text { hind margin of paranota } \\
\text { slightly convex }\end{array}$ & hind margin of paran & list & \\
\hline Tegmina & \multicolumn{3}{|c|}{$\begin{array}{l}\text { In both sexes with black longitudinal band; tegmina of female overlapping dorsally; } \\
\text { tegmina of male converging posteriorly }\end{array}$} & $\begin{array}{l}\text { Tegmina unicolorous yel- } \\
\text { lowish or sometimes a pre- } \\
\text { apical dark brown streak, } \\
\text { tegmina of female overlap- } \\
\text { ping dorsally; tegmina of } \\
\text { male relatively transverse }\end{array}$ \\
\hline $\begin{array}{l}\text { Number of teeth } \\
\text { on male stridula- } \\
\text { tory file }\end{array}$ & $\begin{array}{l}245-300 \\
(\mathrm{CH} 0333, \mathrm{CH} 1507)\end{array}$ & $\begin{array}{l}265 \\
(\mathrm{CH} 3321)\end{array}$ & $\begin{array}{l}255 \\
(\mathrm{CH} 3325)\end{array}$ & $100-120$ \\
\hline $\begin{array}{l}\text { Hind femora } \\
\text { (length in } \mathrm{mm})\end{array}$ & $\begin{array}{l}18-29 ; \text { ventral margin } \\
\text { rarely with } 1-2 \text { small } \\
\text { spinules }\end{array}$ & $\begin{array}{l}\text { ith black dorsal and ventral li } \\
\text { without spinule }\end{array}$ & $\begin{array}{l}21-25.5 \text {; ventral margin } \\
\text { without spinule }\end{array}$ & $\begin{array}{l}\text { 14-18; without black } \\
\text { dorsal and ventral lines; } \\
\text { ventral margin without } \\
\text { spinule }\end{array}$ \\
\hline Male & $\begin{array}{l}\text { see Fig. } 2, \mathrm{~A}-\mathrm{C}, \mathrm{I}, \mathrm{J} \\
\text { Apex produced into an } \\
\text { acute tip; outer margin } \\
\text { with strong teeth }\end{array}$ & $\begin{array}{l}\text { see Fig. 2, D-F } \\
\text { Apex slightly widened or } \\
\text { with subparallel sides, } \\
\text { slightly flattened; outer } \\
\text { margin with small teeth }\end{array}$ & $\begin{array}{l}\text { see Fig. } 2 \text { G, K } \\
\text { Apex slightly widened, } \\
\text { slightly flattened; outer } \\
\text { margin with small teeth }\end{array}$ & $\begin{array}{l}\text { see Fig. } 2, \mathrm{H} \\
\text { Apex widened (spatulate) } \\
\text { and flattened; outer margin } \\
\text { minutely serrate }\end{array}$ \\
\hline $\begin{array}{l}\text { Male subgenital } \\
\text { plate }\end{array}$ & $\begin{array}{l}\text { Posteriorly with very deep } \\
\text { notch; exceeding to cercal } \\
\text { apices; apical part as Fig. } \\
\text { 2, I, J, O }\end{array}$ & $\begin{array}{l}\text { Posteriorly with very } \\
\text { shallow notch or straight; } \\
\text { exceeding to cercal apices; } \\
\text { apical part as Fig. 2, P }\end{array}$ & $\begin{array}{l}\text { Posteriorly with very } \\
\text { shallow notch or straight; } \\
\text { not reaching the cercal api- } \\
\text { ces; apical part as Fig. 2, } \\
\text { K, Q }\end{array}$ & $\begin{array}{l}\text { Posteriorly with very } \\
\text { shallow notch or straight; } \\
\text { much shorter or exceeding } \\
\text { to cercal apices; apical part } \\
\text { as Fig. 2, L-N, R }\end{array}$ \\
\hline $\begin{array}{l}\text { Female subgenital } \\
\text { plate }\end{array}$ & \multicolumn{2}{|c|}{ Short, distinctly transverse } & $\begin{array}{l}\text { Short, distinctly transverse; } \\
\text { sometimes with median } \\
\text { carinae }\end{array}$ & $\begin{array}{l}\text { Short, distinctly transverse; } \\
\text { sometimes with small } \\
\text { process at hind margin }\end{array}$ \\
\hline Ovipositor (mm) & $8.5-12.5$ & $10-11.6$ & $10.8-11.2$ & $5.5-7.2$ \\
\hline $\begin{array}{l}\text { Gonangulum and } \\
\text { Lamella }\end{array}$ & $\begin{array}{l}\text { Gonangulum widened dor- } \\
\text { soventrally, relatively } \\
\text { swollen, forming a deep } \\
\text { vertical pit with lamella; } \\
\text { lamella strong and slightly } \\
\text { swollen }\end{array}$ & $\begin{array}{l}\text { Gonangulum with round } \\
\text { edges, slightly widened } \\
\text { dorsoventrally, not } \\
\text { swollen; lamella with wide } \\
\text { surface, not folding, } \\
\text { forming with gonangulum } \\
\text { a large, but not deep pit }\end{array}$ & $\begin{array}{l}\text { Gonangulum with round } \\
\text { edges, slightly widened } \\
\text { dorsoventrally, not } \\
\text { swollen; lamella with wide } \\
\text { surface, slightly folding, } \\
\text { forming with gonangulum } \\
\text { a large, but not deep pit }\end{array}$ & $\begin{array}{l}\text { Lamella of dorsal margin } \\
\text { of lower ovipositor valve } \\
\text { lamelliform, moderately } \\
\text { extending laterally, but } \\
\text { strongly impressed dorsally } \\
\text { forming with gonangulum } \\
\text { a round, deep and dorso- } \\
\text { laterally facing pit }\end{array}$ \\
\hline
\end{tabular}


TABLE 2A. Measurements of $P$. sanctipauli (length in mm; $\mathrm{SD}$ - standard deviation)

\begin{tabular}{llccrl}
\hline & (Turkey only) & $\mathrm{n}$ & range & mean & SD \\
\hline Male & Body & 29 & $20.2-31.0$ & 24.4 & 2.4 \\
& Pronotum & 29 & $5.6-8.8$ & 7.2 & 0.7 \\
& Tegmina & 29 & $3.0-4.5$ & 3.6 & 0.3 \\
& Hind femur & 28 & $18.0-25.0$ & 21.1 & 1.5 \\
\hline Female & Body & 31 & $22.3-32.4$ & 26.5 & 2.6 \\
& Pronotum & 31 & $6.5-8.8$ & 7.7 & 0.6 \\
& Tegmina & 29 & $1.4-3.7$ & 2.6 & 0.4 \\
& Hind femur & 30 & $20.0-28.8$ & 23.3 & 1.9 \\
& Ovipositor & 30 & $9.1-11.0$ & 10.1 & 0.5 \\
\hline
\end{tabular}

TABLE 2B. Measurements of $P$. sanctipauli (length in $\mathrm{mm}$ ).

\begin{tabular}{lllll}
\hline Hindfemur & & Turkey & Rhodos & Samos \\
\hline Male & range & $18.0-25.0$ & $18.5-21.0$ & $20.5-21.5$ \\
& Mean & 21.1 & 19.8 & 21.1 \\
& $\mathrm{n}$ & 28 & 12 & 6 \\
\hline Female & range & $20.0-28.8$ & $19.5-23.5$ & 23.5 \\
& Mean & 23.3 & 22.3 & \\
& $\mathrm{n}$ & 30 & 10 & 1 \\
\hline
\end{tabular}

scraper moves along the long outer part of the file, then probably stops at the bulge (Fig. 5), and finally moves along the short proximal part of the file. Since the song is difficult to characterise by measurements, an example of the intra-population variability in amplitude modulation is shown in Fig. 9. In the recordings of 15 animals the twopart syllable structure is easily recognised. The duration of the syllable depends on temperature and is about 500 $\mathrm{ms}$ at $25^{\circ} \mathrm{C}$. In the course of the afternoon the syllables become longer and of the morning shorter (Fig. 9).

A female responds to male song by abruptly closing her tegmina, producing one or a few impulses (Heller \& Helversen, 1986). A schematic figure of the position of the non-homologous stridulatory organs in males and females of $P$. sanctipauli can be found in Heller \& Helversen (1986).

The frequency spectrum of the song (analysed up to 70 $\mathrm{kHz}$ ) has a distinct maximum in the high audio range between 10 and $20 \mathrm{kHz}$ (Heller \& Helversen, 1986; Heller, 1988).

Material examined. GREECE: Aegean Islands: N. Samos, Samos: $1 \mathrm{~km}$ westl. Vathi $\left(26^{\circ} 57^{\prime} \mathrm{E} ; 37^{\circ} 44^{\prime} \mathrm{N}\right)$, leg. Jay McCartney, 19.v.1998, $10^{\star} ; 3 \mathrm{~km}$ nordöstl. Mytilinioi $\left(26^{\circ} 54^{\prime} \mathrm{E}\right.$; $\left.37^{\circ} 44^{\prime} \mathrm{N}\right)$, leg. Heller \& Volleth, 19.v.1998, 1 ơ $^{\text {; }} 3 \mathrm{~km}$ südlich Pirgos $\left(26^{\circ} 47^{\prime} \mathrm{E} ; 37^{\circ} 41^{\prime} \mathrm{N}\right)$, leg. Heller \& Volleth, 20.v.1998, $10^{\circ}, 1$ \% ; Moni Vronta $\left(26^{\circ} 51^{\prime} \mathrm{E} ; 37^{\circ} 47^{\prime} \mathrm{N}\right)$, leg. K.-G. Heller \& M. Volleth, 21.v.1998, $1 \delta^{\text {* }}$; Psili Ammos südl. Vathy $\left(27^{\circ} 0^{\prime} \mathrm{E}\right.$; $\left.37^{\circ} 42^{\prime} \mathrm{N}\right)$, leg. Heller \& Volleth, 19.v.1998, 1 o $^{\star}$; Psili Ammos südl. Vathy $\left(27^{\circ} 0^{\prime} \mathrm{E} ; 37^{\circ} 42^{\prime} \mathrm{N}\right)$, leg. Jay McCartney, 19.v.1998, $1 \delta^{\star}$; Umg. Kallithea $\left(26^{\circ} 34^{\prime} \mathrm{E}\right.$; $\left.37^{\circ} 44^{\prime} \mathrm{N}\right)$, leg. Heller \& Volleth, 21.v.1998, 20, 1\%; Southern Sporadhes: N. Rhodos: Rhodos, leg. A. Stumpner, 1.-31.iv.1987, 10; Dimilia $\left(28^{\circ} 0^{\prime} \mathrm{E}\right.$; $36^{\circ} 16^{\prime} \mathrm{N}$ ), elev. $30 \mathrm{~m}$, leg. Heller, 14.-15.iv.1983, $20^{\star}, 1$ 우 (see Heller, 1984b); Embonas $\left(27^{\circ} 51^{\prime} \mathrm{E}\right.$; $\left.36^{\circ} 12^{\prime} \mathrm{N}\right)$, leg. Heller, 10.-24.iv.1983, $1 \delta^{\widehat{ }}$ (see Heller, 1984b); Lindos $\left(28^{\circ} 4^{\prime} \mathrm{E}\right.$; $36^{\circ} 5^{\prime} \mathrm{N}$ ), elev. $30 \mathrm{~m}$, leg. Heller, 18.-19.iv.1983, $12 \delta^{\hat{0}}, 10$ 우 (see

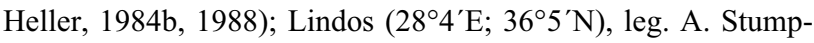
ner, 20.iv.1987, 2 \%. TURKEY: Aydın: Çine, Bölüntü

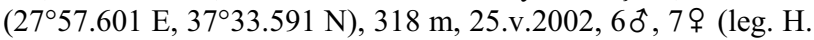
Sevgili \& Y. Durmus); Bölüntü, 6.vi.1950, 1 \% (leg. I. Tuncay) (see Karabag 1958); Gözkayası, leg. G. Hayretdag, 2.vi.1967, 10 , 1 ㅇ (see Karabag et al., 1971); Merkez, Savrandere between Aydın and Çine, 11.v.1976, 1 б, 7 ㅇ (AZME; see Karabag et al., 1981); Denizli: Akyar südwestl. Denizli $\left(29^{\circ} 10^{\prime} \mathrm{E}\right.$; $\left.37^{\circ} 37^{\prime} \mathrm{N}\right)$, leg. Heller, 6.v.1985, 20 (CH); Denizli-Antalya yolu, $18 . \mathrm{km}$ (Yol kenarı, Orman Fidanlıg1), 27.v.2002, $2 \hat{\delta}$ (1 $\hat{\delta}$ in alcohol) (leg. H. Sevgili); Izmir: Menemen, 10.v.1950, 1 ㅇ (leg?); Belevi nordöstl. Selcuk $\left(27^{\circ} 27^{\prime} \mathrm{E} ; 38^{\circ} 1^{\prime} \mathrm{N}\right)$, leg. Heller, 28 April 1985, $10^{\star}, 3$ ㅇ $(\mathrm{CH})$; Ödemis, Bozdag $\left(28^{\circ} 02.951 \mathrm{E}, 38^{\circ} 18.798 \mathrm{~N}\right)$, 520 m, 25.v.2002, 30 , 1 ㅇ (leg. H. Sevgili \& Y. Durmus); Manisa: Kürkçü, 4.vi.1940, 3 đે, 1 @ (leg?) (AZME); Gölmarmara, Yeniköy, $38^{\circ} 47.858$ N $28^{\circ} 04.432 \mathrm{E}, 447 \mathrm{~m}$, 24.v.May 2002, 3 के (leg. H. Sevgili \& Y. Durmus); Mugla: Labranda $\left(27^{\circ} 42^{\prime} \mathrm{E} ; 37^{\circ} 24^{\prime} \mathrm{N}\right)$, leg. Heller, 2.v.1985, 3 $\delta^{\wedge}, 2$ 우 (CH); Köyceiz, Topraksu Tesisleri, 1.v.1974, $10^{\Uparrow}$ (see Karabag et al., 1981); Datça, Sınd1, 9.v.1975, 1 ㅇ (AZME; see Önder et al., 1999); Gökova, Marmaris, 29.v.1985, 1 đิ (leg. B. Gürkan); Güllük $\left(27^{\circ} 36^{\prime} \mathrm{E} ; 37^{\circ} 14^{\prime} \mathrm{N}\right)$, leg. M. Gebhardt, 6.iv.1987, $2 \delta^{\dagger}$, 1 ㅇ (CH; see Heller, 1988); Düzeyin (Oyuklu dagi, Y1lanlı) 30.vi.1987, 10ð , 9 ㅇ (leg. A. Demirsoy); Marmaris, Turunç, 4.vi.2000, 60ิ, 15 9 ; Marmaris Milli Park1, Sogukbelen tepesi, 14.vi.1997, 1ठ; Marmaris Milli Parký, Kumbükü mevki, 18.v.1997, 1ठ; Marmaris Milli park1, Turunç, Dereözü mah., 320 m, 18.v.1997, 1 ô, 1 \% ; Marmaris, Adaagzı, Günlük orman1, 3.vi.2000, $2 \sigma^{\star}$ (leg. Y. Durmus); pass near Ula (ca. $6 \mathrm{~km} \mathrm{~s}$ Mugla) $\left(28^{\circ} 22^{\prime} \mathrm{E} ; 37^{\circ} 5^{\prime} \mathrm{N}\right)$, elev. $700 \mathrm{~m}$, leg. K.-G. Heller, 10.vi.2000, $1 \delta^{\dagger}(\mathrm{CH})$ (all except specimens from Greece or marked with $\mathrm{CH}$ or AZME in HUZOM).

Previous records (see also Material examined). GREECE: Aegean Islands: Samos: Marathokombas Werner, 1933 (BeyBienko 1954; Harz, 1969; Willemse, 1982, 1984); Kos: Kos, Ramme, 1933 (Harz, 1969; Willemse, 1982, 1984); Mt. Dikeo Werner, 1936 (Willemse, 1982, 1984); Kalimnos: Potea Werner 1936 (Bey-Bienko, 1954; Willemse, 1982, 1984); Nisyros: SysTax/DORSA: ZMHB, 2 \%, leg. v. Oertzen; Rhodos: Rhodos Brunner von Wattenwyl, 1878, Ramme, 1933 (Bey-Bienko, 1954; Harz, 1969; Willemse, 1982, 1984), Heller \& Helversen, 1986; Lindos Jannone, 1936; Willemse, 1982 (Willemse, 1984); A. Isidoros Giglio-Tos, 1914 (Willemse, 1982, 1984); surroundings Rodhini, several localities Werner, 1936 (Willemse, 1982, 1984); Ag. Irini near Apolakia Heller, 1984b; Feraklos Heller, 1984b; Simi: Pedhion Willemse, 1984; TURKEY: Antalya: Akseki, Bademli, 1550 m, leg. A. Koçak, 22.vi.1991, 40ิ, 1 \%: Ünal, 1999; Aydın: Aydın Werner, 1901 (Ramme, 1933), BeyBienko, 1954); Priene Heller, 1988; Germencik Önder et al., 1999; Izmir: Efes (= Ephesus) Brunner von Wattenwyl, 1878, Werner, 1901; Bei-Bienko, 1954, (Ramme, 1933; Karabag, 1958); Kis Avle (Smyrna; ex Coll. Lederer) (paratypes in NHMW; not localised); Göztepe Werner, 1901 (Ramme, 1933; Karabag, 1958); Sewdiköi Ramme, 1933; Boz Dag Ramme, 1933; Izmir, v.1912 (leg. de Kerville) Chopard, 1939 (Karabag, 1958); Bayındır; Bergama; Dikili; Kemalpasa; Seferihisar; Selçuk; (leg. E. Tazegül) all Tazegül \& Önder, 1995; Manisa, Alasehir (leg. Werner) Werner, 1901, Bei-Bienko, 1954 (Karabag, 1958); Mugla: Fethiye (= Makri) Ramme, 1933 (to P. pulcher?) (Karabag, 1958); Mugla (leg. M. Burr) Karabag, 1958; the island of Chios (Greece) is mentioned by Bey-Bienko (1954) but not in the references where this information is said to be in (Ramme, 1933; Werner, 1936).

Distribution. West Anatolia and the Greek islands Rhodos, Nisyros, Kos, Samos, Kalymnos and Simi. The record from Akseki/Antalya (leg. A. Koçak; Ünal, 1999) needs confirma- 


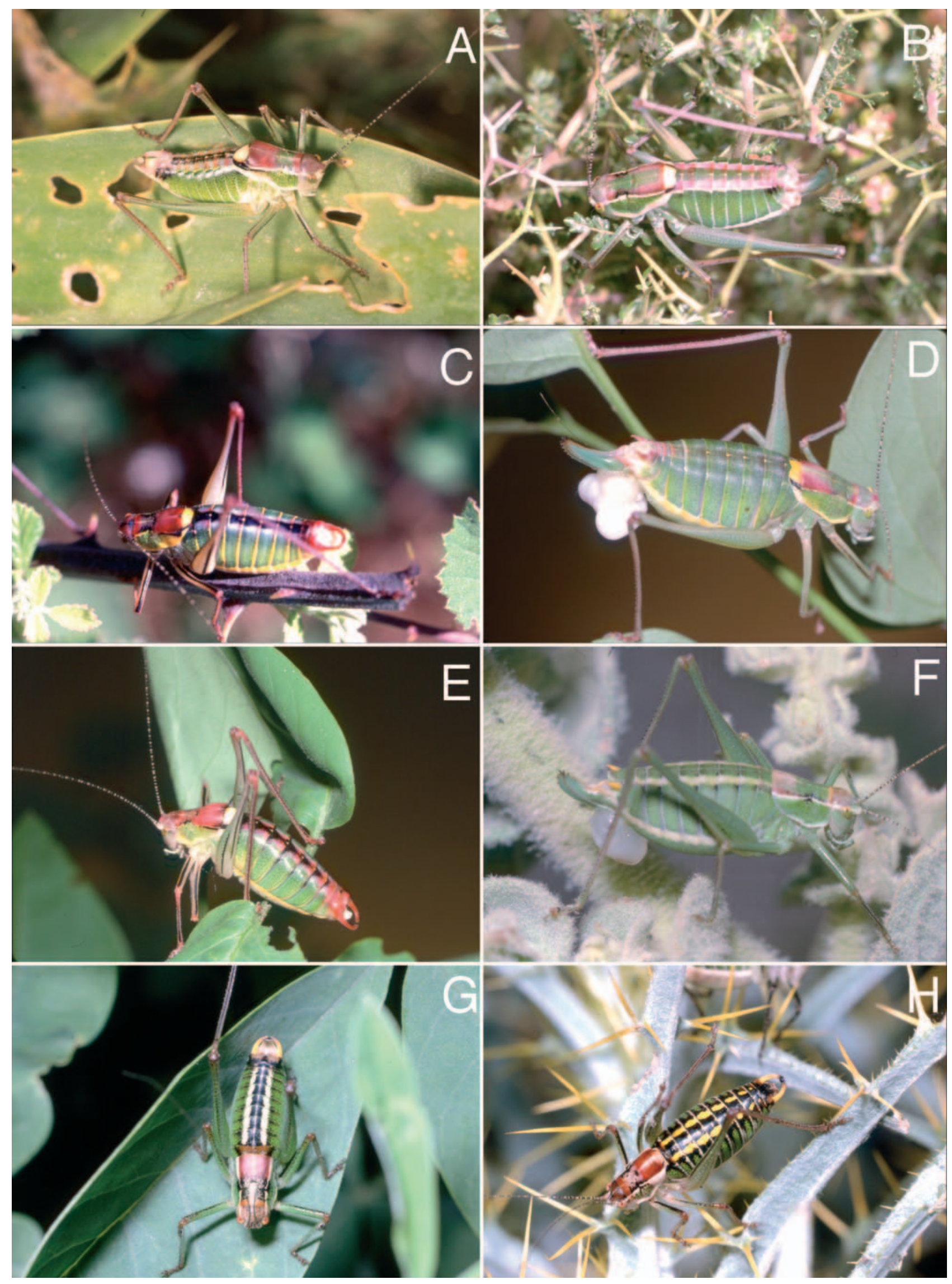

Fig. 1. A - Poecilimon sanctipauli, male (Rhodos, Lindos); B - P. sanctipauli, female (Samos, Kallithea); C - P. lodosi, male (Manisa); D - P. lodosi, female (Manisa); E - P. pulcher, male (Aydin, Söke); F - P. mytilenensis brevissimus, female (Lesbos, Moni Limonos); $\mathrm{G}$ - P. mytilenensis mytilenensis, male (Lesbos, Rachidi); H - P. mytilenensis brevissimus, male (Lesbos, Agra). 

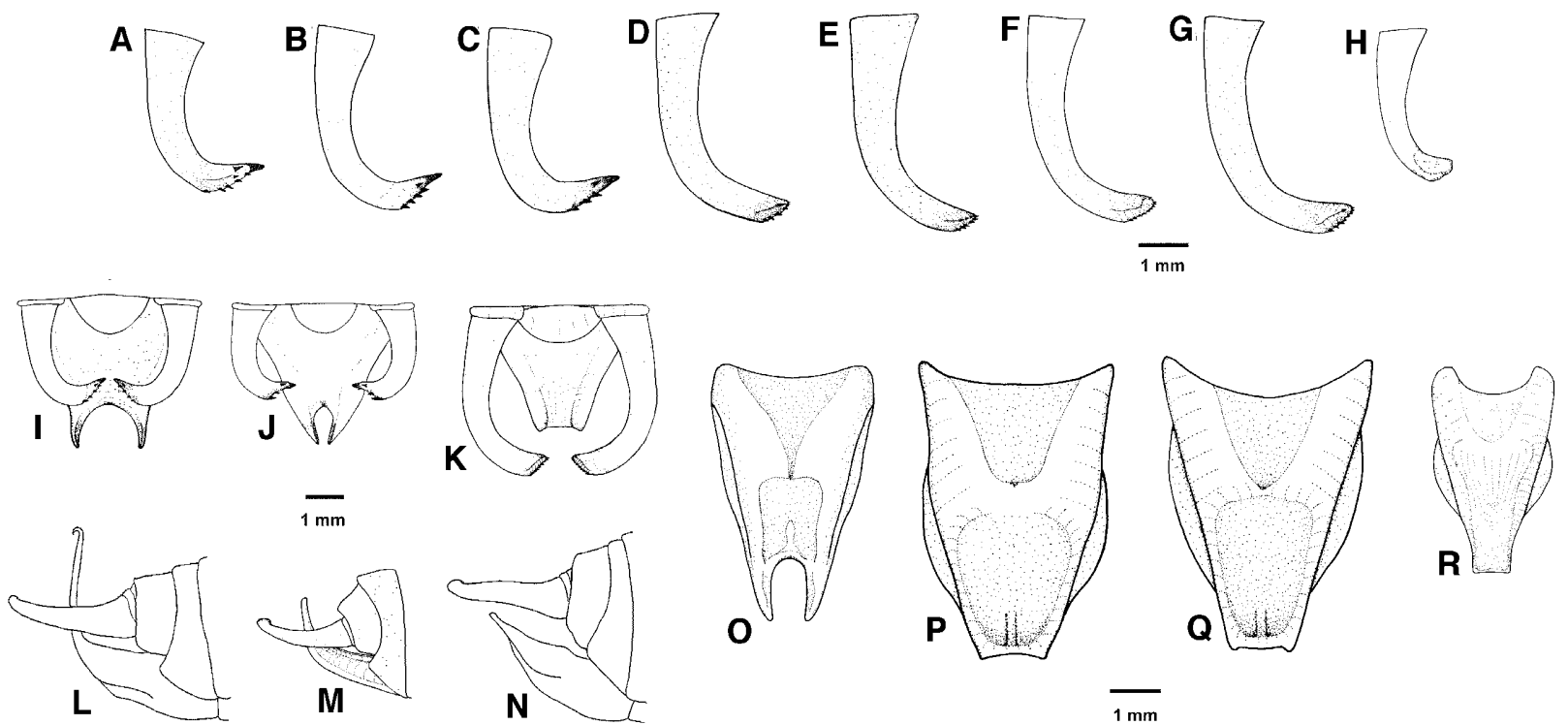$$
\mathbf{N}
$$

Fig. 2. Structures of male genitalia. A-H - left cercus: A, B - P. sanctipauli, Aydin, Çine, Bölüntü; C - P. sanctipauli, Manisa, Gölmarmara, Yeniköy; D, E - P. pulcher, Izmir, Ödemis; F - P. pulcher, Izmir, Cesme; G - P. lodosi, Manisa; H - P. mytilenensis, Ayvalik, Alibey island; I-K - Cerci with subgenital plate, dorsal view: I - P. sanctipauli, Izmir, Bozdagh; J - P. sanctipauli Denizli, Antalya yolu, $18^{\text {th }} \mathrm{km} ; \mathrm{K}-P$. lodosi, Manisa; L-N - Cerci with subgenital plate, lateral view: $\mathrm{L}-P$. mytilenensis mytilenensis (Greece, Lesvos, Ayiassos); M - P. mytilenensis mytilenensis Turkey, Ayvalik, Alibey island; N - P. mytilenensis brevissimus (Greece, Lesvos, 3 km NW Kaloni); O-R - subgenital plate, ventral view: O - P. sanctipauli Manisa, Gölmarmara, Yeniköy; P - P. pulcher Izmir, Ödemis; Q - P. lodosi Manisa; R - P. mytilenensis, Ayvalik, Alibey island.

TABle 3A. Measurements of $P$. pulcher (length in mm). Body dimensions of types not correct due to stretching/ shrinkage according to Ramme (1933: 536-7).

\begin{tabular}{|c|c|c|c|c|c|}
\hline \multicolumn{2}{|c|}{$\begin{array}{l}\text { all specimens } \\
\text { except those from } \\
\text { Izmir, Çesme }\end{array}$} & $\mathrm{n}$ & range & mean & $\begin{array}{l}\text { lectotype/allotype } \\
\text { (Brunner 1891 / } \\
\text { Ramme, 1933) }\end{array}$ \\
\hline Male & Body & 4 & $30.3-32.7$ & 31.8 & $30 / 29$ \\
\hline & Pronotum & 6 & $8.9-9.5$ & 9.2 & 8.7 \\
\hline & Tegmina & 3 & $3.1-3.9$ & 3.4 & \\
\hline & Hind femur & 5 & $23.5-26.5$ & 25.2 & 24 \\
\hline \multirow[t]{5}{*}{ Female } & Body & 1 & 30.6 & & $28 / 26$ \\
\hline & Pronotum & 4 & $8.7-10.0$ & 9.4 & \\
\hline & Tegmina & 1 & 2.8 & & \\
\hline & Hind femur & 4 & $24-27.5$ & 25.9 & 21.5 \\
\hline & Ovipositor & 4 & $11.5-12.5$ & 11.9 & 10 \\
\hline
\end{tabular}

TABle 3B. Measurements of $P$. pulcher (length in $\mathrm{mm}$ ).

\begin{tabular}{llccc}
\hline \multicolumn{2}{l}{ Izmir, Çesme } & $\mathrm{n}$ & range & mean \\
\hline Male & Body & 4 & $23.3-24.0$ & 23.8 \\
& Pronotum & 4 & $7.3-7.7$ & 7.5 \\
& Tegmina & 3 & $2.7-3.0$ & 2.8 \\
& Hind femur & 4 & $20.7-21.3$ & 21.0 \\
Female & Body & 2 & $22.0-23.3$ & 22.7 \\
& Pronotum & 2 & $7.5-7.8$ & 7.7 \\
& Tegmina & 2 & $1.8-2.3$ & 2.1 \\
& Hind femur & 1 & 21.2 & \\
& Ovipositor & 1 & 10.2 & \\
\hline
\end{tabular}

tion. It is a long way from the other localities and we were unable to check the specimens deposited in the Centre of Entomological Studies, Ankara.

\section{Poecilimon pulcher Brunner von Wattenwyl, 1891}

Poecilimon pulcher Brunner von Wattenwyl, 1891: 25.

Type locality. Turkey: Bozdag near Izmir (Smyrna).

Type depository. NHMW, lectotype $\hat{0}$ (from syntypes 10 , 1 \%; male collected by Lederer, female (without locality label) by Türk in 1870; information provided by A. Kaltenbach, specimens not seen). According to the Code ( $\$ 74.5$; ICZN 1999) the comments of Ramme (1933: 536) "Ich wähle neben dem Holotypus (Unicum) des Männchens..." (In addition to the male holotype (unicum) I select...) are sufficient for a lectotype designation.

P. pulcher: Brunner von Wattenwyl, 1891: 25 (key), 27-28 (description); Jacobson 1905: 330 (key), 356 (description; in Russian); Kirby, 1906: 376 (catalogue); Ramme, 1933: 507, 509 (arrangement), 510 (checklist), 536 (description); Bey-Bienko, 1954: 256 (key), 287-8 (description); Karabag, 1958: 82 (faunistic catalogue); Tazegül \& Önder, 1995: 3 (distribution); Önder et al., 1999: 247 (distribution); Çıplak et al., 1999: 753 (checklist, distribution); Çıplak et al., 2002: 685 (checklist, distribution).

Databank: SysTax, OSF2.

Diagnosis/description. See Table 1, 3 and Figs 1-4.

Song. The basic structure of the song is very similar to that of $P$. sanctipauli (isolated syllables produced at intervals of several seconds). However, the duration of the syllables is much longer (at $25^{\circ} \mathrm{C}$ often more than one second; Fig. 7).

Material examined. TURKEY: Aydin: Dilek Yarimadasi M. P. (ca. $20 \mathrm{~km}$ wsw Söke) $\left(27^{\circ} 12^{\prime} \mathrm{E} ; 37^{\circ} 42^{\prime} \mathrm{N}\right)$, elev. $5 \mathrm{~m}$, leg. K.-G. Heller, 11.vi.2000, $20^{\star}, 2{ }^{\circ}$; $\left(27^{\circ} 5^{\prime} \mathrm{E} ; 37^{\circ} 40^{\prime} \mathrm{N}\right)$, leg. 

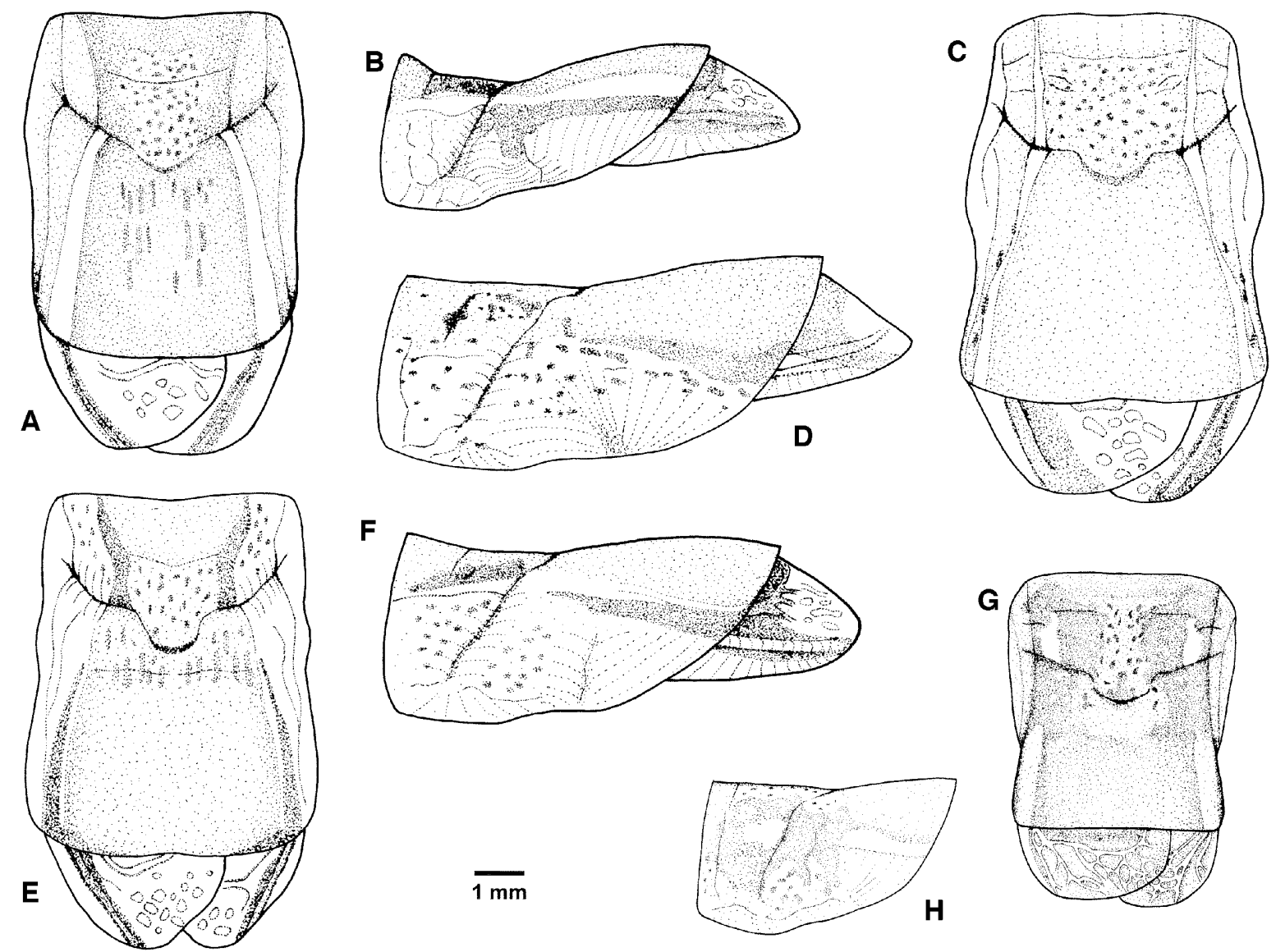

Fig. 3. Male pronotum, dorsal and lateral view. A, B - P. sanctipauli: A - Manisa, Gölmarmara, Yeniköy; B - Aydin, Çine, Bölüntü; C, D - P. pulcher, Izmir, Ödemis; E, F - P. lodosi, Manisa; G, H - P. m. mytilenensis, Ayvalik, Alibey island.

Heller, 3.v.1985, 1 ㅇ (CH); Izmir, Ödemis, Çaglayan köyü $\left(27^{\circ} 58.032 \mathrm{E}, 38^{\circ} 05.030 \mathrm{~N}\right), 421 \mathrm{~m}, 25 . \mathrm{v} .2002,5 \delta^{\hat{0}}, 1$ 우 $\left(1 \hat{\sigma}^{\hat{\mathrm{t}}}\right.$ in alcohol, on Umbellifera) (leg. H. Sevgili \& Y. Durmus); Çesme, 1951, 40, 2 ㅇ (leg. Izmir Zirai Mücadele Enst.); Menemen, 10.v.1950, 2 q (leg.?, det. T. Karabag, 1950), all HUZOM.Previous records: TURKEY: Izmir: Bozdag, $\delta$, + , Brunner von Wattenwyl, 1891 (Bei-Bienko, 1954); Narlıdere, (Bornova Mücadele Enstitüsü) Karabag, 1958; Çesme, SysTax/DORSA: ZMUH (1 ô, 1ㅇ, v.1951, leg. T. Karabag); Aliaga; Bergama; Kemalpasa; Menemen; Ödemis; Seferihisar; Selçuk; Tire, Torbalı, (leg. E. Tazegül) Tazegül \& Önder, 1995; Bergama, Menemen, both Önder et al., 1999. The localities Aydın, Göztepe,

TABle 4. Measurements of $P$. lodosi (length in $\mathrm{mm}$; SD standard deviation).

\begin{tabular}{llcccc}
\hline & & $\mathrm{n}$ & range & mean & SD \\
\hline Male & Body & 7 & $28.0-32.7$ & 29.6 & 1.9 \\
& Pronotum & 17 & $7.2-8.5$ & 7.9 & 0.4 \\
& Tegmina & 6 & $2.3-3.5$ & 3.0 & 0.5 \\
& Hind femur & 16 & $21.8-24$ & 22.8 & 0.7 \\
\hline Female & Body & 6 & $27.2-31.3$ & 29.0 & 1.5 \\
& Pronotum & 21 & $7.3-9.1$ & 8.5 & 0.4 \\
& Tegmina & 5 & $2.8-3.8$ & 3.2 & 0.4 \\
& Hind femur & 21 & $22.5-26.0$ & 24.6 & 0.8 \\
& Ovipositor & 20 & $9.0-11.2$ & 10.3 & 0.7 \\
\hline
\end{tabular}

Efes and Seydiköy mentioned by Karabag (1958) based on Ramme (1933) refer to $P$. sanctipauli.

Distribution. Known from the region around Izmir, western Anatolia.

\section{Poecilimon lodosi Harz, 1975}

Poecilimon lodosi Harz, 1975: 9.

Type locality. Turkey: Manisa.

Type depository. MHNG, holotype $\delta$ (not seen).

P. lodosi: Harz, 1975: 9 (description); Otte, 1997: 124 (catalogue); Önder et al., 1999: 247 (distribution); Naskrecki \& Otte, 1999: (catalogue); Çıplak et al., 1999: 753 (checklist, distribution); Çıplak et al., 2002: 686 (checklist, distribution).

Databank: OSF2.

Diagnosis/description. See Table 1, 4 and Figs 1-4.

It does not clearly differ morphologically from $P$. pulcher.

Song. The song is very similar to that of $P$. sanctipauli (see Fig. 7), also in syllable duration. The songs of some animals reared in the laboratory and in permanent acoustical contact with other males sometimes contained irregular intra-syllable gaps and impulse series after the second part of the syllable, perhaps some kind of rivalry song. Females responded to male songs in the same way as those of $P$. sanctipauli.

Material examined. TURKEY: Manisa: Sipil Dagh (ca. $3 \mathrm{~km}$ südl. Manisa) $\left(27^{\circ} 26^{\prime} \mathrm{E} ; 38^{\circ} 34^{\prime} \mathrm{N}\right)$, elev. $600 \mathrm{~m}$, leg. K.-.G. 

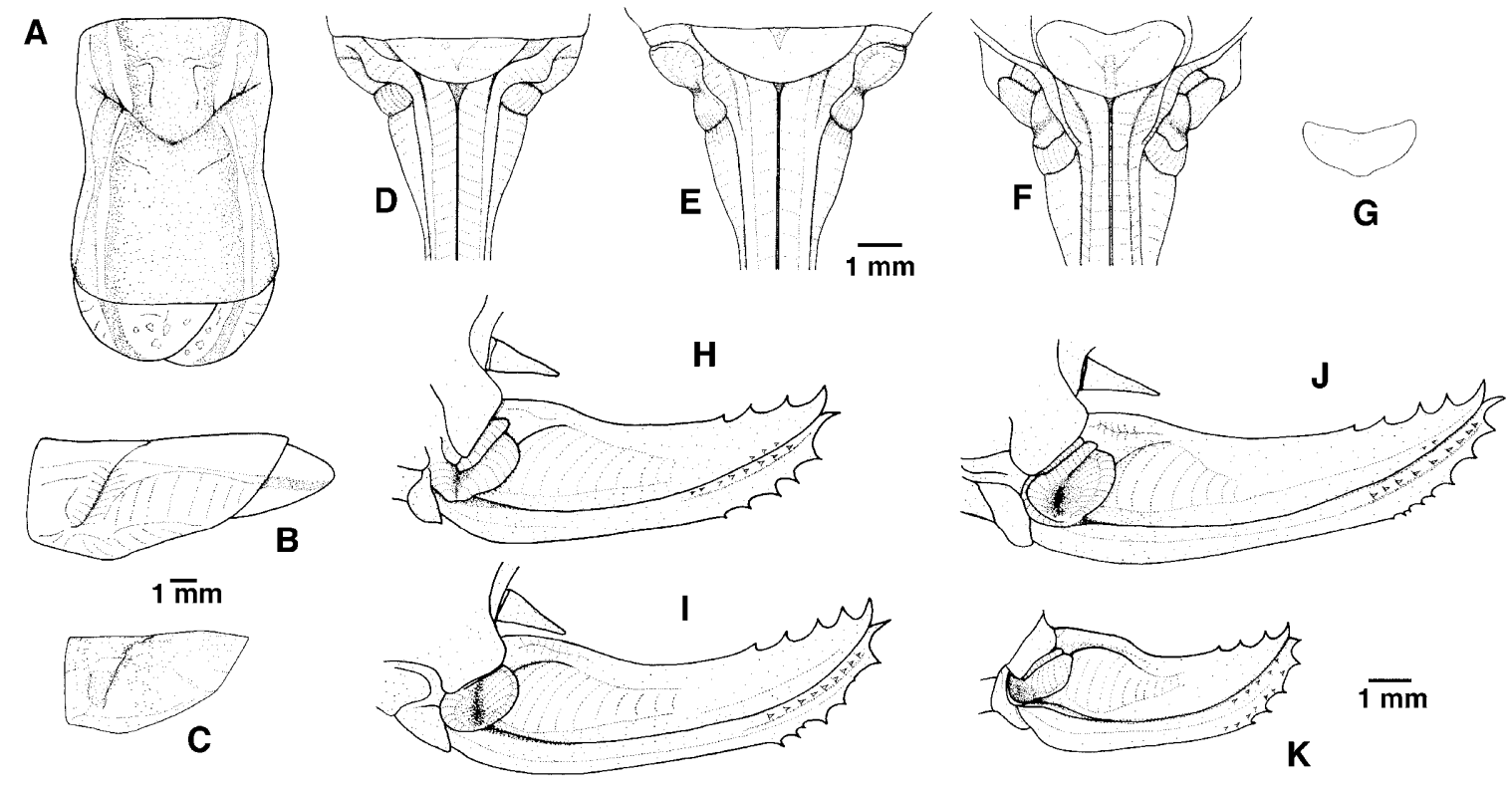

Fig. 4. Female morphological structures. A - pronotum, dorsal view: $P$. lodosi, Manisa; $\mathrm{B}-\mathrm{C}-$ pronotum lateral view: $\mathrm{B}-P$. lodosi, Manisa; C - P. m. mytilenensis, Ayvalik; D-F - base of ovipositor and subgenital plate: D - P. sanctipauli Mugla, Marmaris; $\mathrm{E}-P$. pulcher, Izmir, Ödemis; $\mathrm{F}$ - P. lodosi, Manisa; $\mathrm{G}$ - subgenital plate P. mytilenensis, Ayvalik; $\mathrm{H}-\mathrm{K}$ - ovipositor: $\mathrm{H}-P$. sanctipauli Mugla, Marmaris; I - P. pulcher, Izmir, Ödemis; J - P. lodosi, Manisa; K - P. mytilenensis, Ayvalik.

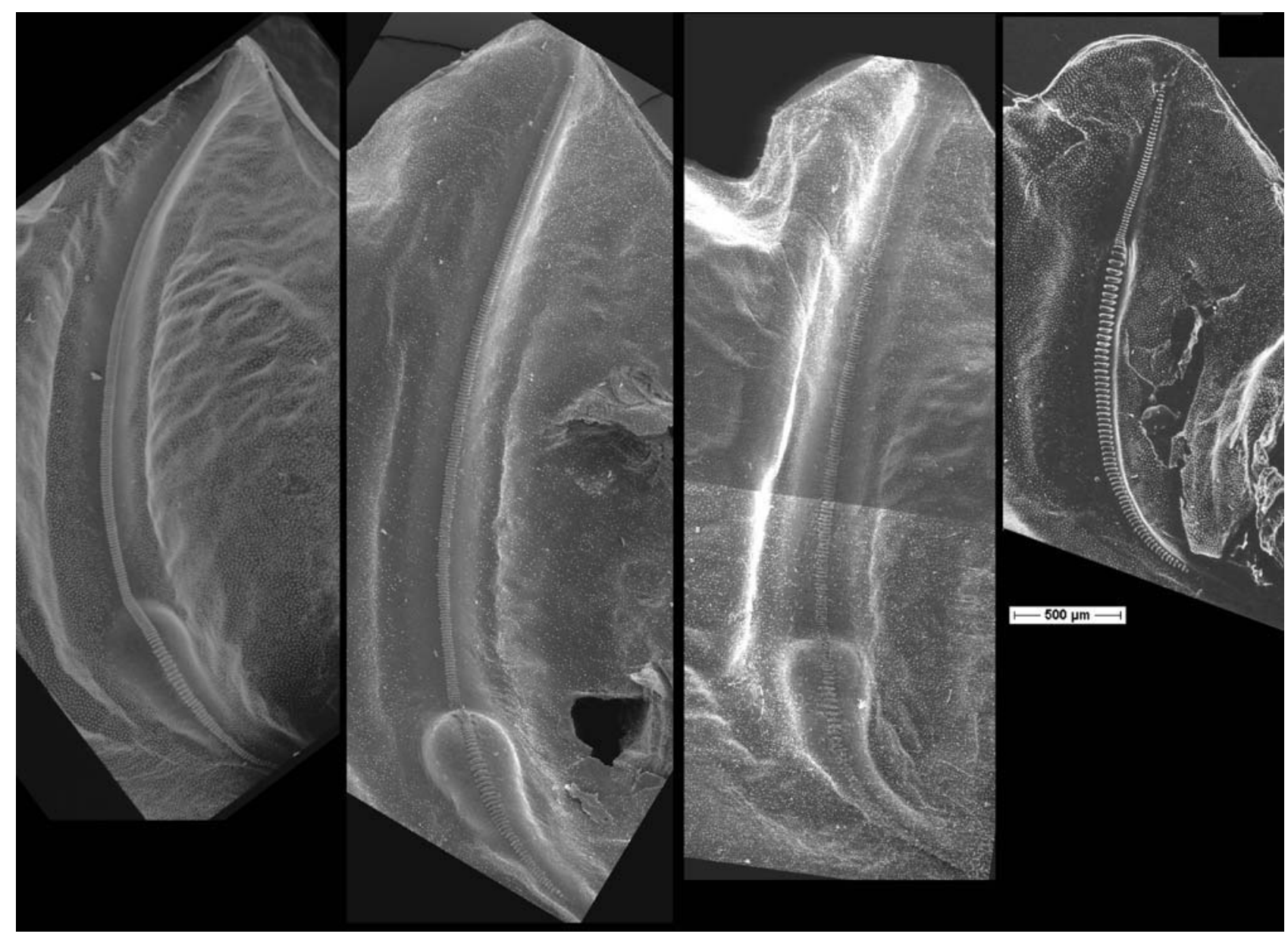

Fig. 5. Male stridulatory file, tegmen articulation at the bottom. A - P. sanctipauli Rhodos, Dimilia CH0333; B - P. pulcher Aydin, Söke CH3321; C - P. lodosi, Manisa, CH3325; D - P. m. mytilenensis Lesvos, Ayiassos. 

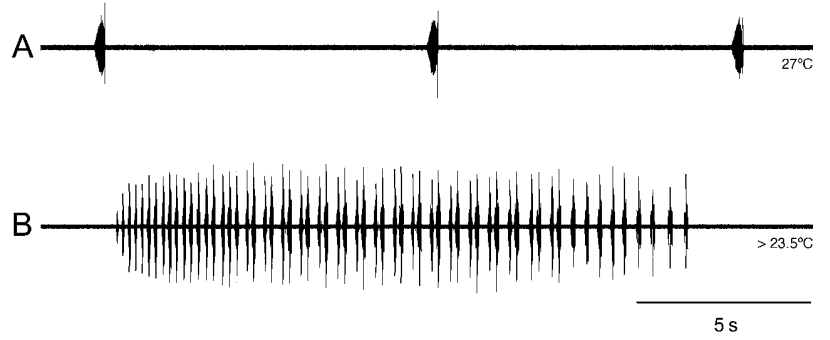

Fig. 6. Oscillograms of male calling song. A - P. sanctipauli Samos, Pirgos CH4695; B - P. m. mytilenensis Lesbos, Moria, CH3152.

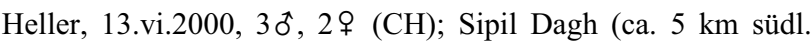
Manisa) $\left(27^{\circ} 26^{\prime} \mathrm{E} ; 38^{\circ} 33^{\prime} \mathrm{N}\right)$, elev. $1200 \mathrm{~m}$, leg. K.-G. Heller, $13 \mathrm{vi.2000}, 10,1 \%$ and offspring of these animals, 1.i.-30.vi.2001, 60ิ, 12 ㅇ (CH); Sipil Dagh $\left(27^{\circ} 25.737\right.$ E, $38^{\circ} 35.297$ N), 717 m, 24.v.2002, 80 , 6 ㅇ (leg. H. Sevgili \& Y. Durmus) (HUZOM).
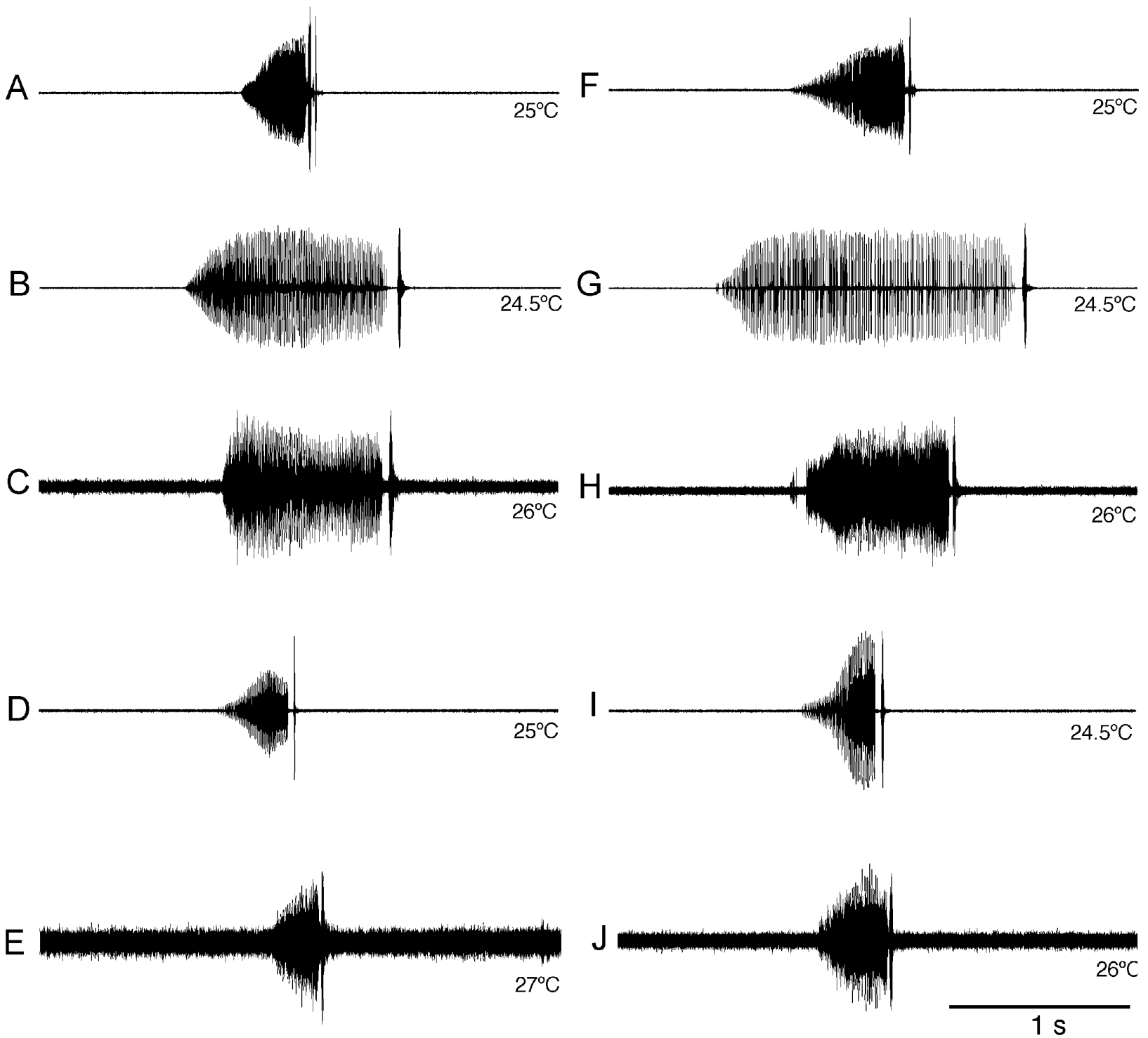

Fig. 7. Oscillograms of male calling song. A-B - P. sanctipauli (A - Denizli, Antalya yolu; B - Ödemis, Bozdag); C-F - P. pulcher (C, D - Izmir, Ödemis, two different males; E, F - Aydin, Söke, two different males); G-J - P. lodosi (Manisa, four different males). 

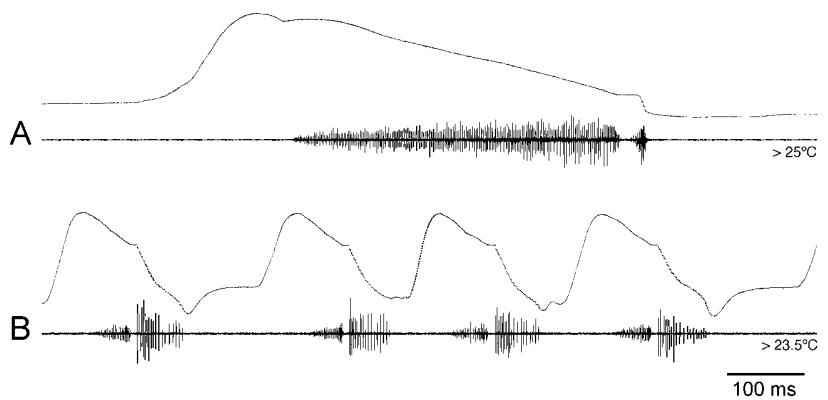

Fig. 8. Oscillograms of stridulatory movements and song [synchronous registration of left tegmen movement (upward deflection represents opening, downward closing) and sound]. A - P. sanctipauli Mugla, Güllük CH2331; B - Poecilimon m. mytilenensis Lesbos, Moria, CH3152.

P. mytilenensis brevissimus Heller, Willemse \& Sevgili, 2005: (in press)

Type locality: Greece, Lesbos (Mytilene), Mithymna.

Type depository: $\mathrm{CH}$, holotype ${ }^{\top}$.

This species was revised by the authors together with Fer Willemse (Heller et al., 2005). Details of the synonymy and localities can be found in that paper.

Diagnosis/description. See Table 1 and Figs 1-4.

Song. In both subspecies the male calling song does not consist of isolated syllables as in the other three species, but of sequences of 25-120 syllables with a duration of 10-30 s (Fig. 6; rarely as short as $6 \mathrm{~s}$; all data recorded at ca. $\left.25^{\circ} \mathrm{C}\right)$. Each syllable has two parts, which are more equal in duration than in $P$. sanctipauli (Fig. 8) Correspondingly the bulge in the stridulatory file is situated near the middle (Fig. 5). Females respond acoustically to male song (see Heller et al., 2005 for details).

Distribution. Islands of Lesbos, Greece, and Alibey, Turkey.

\section{DISCUSSION}

\section{Phylogeny}

The three large species $P$. pulcher, $P$. lodosi and $P$. sanctipauli form a monophyletic group according to their morphological similarity and unique, derived type of bioacoustical signals. Making the additional assumption that a broad or even distally widened cercus apex (Fig. 2 $\mathrm{D}-\mathrm{H})$ in the male is an autapomorphic character of the group results in the following phylogenetic tree will: $[P$. mytilenensis (P. pulcher, P. lodosi, P. sanctipauli)]. However, there are several alternative relationships for the three large species. The form of the cerci and subgenital plate of $P$. sanctipauli and the extremely slow stridulatory movement of $P$. pulcher can be considered as autapomorphic characters. In $P$. lodosi both these characters are in a plesiomorphic state. This species can be grouped with either of the two derived species, and can be considered a relict of the ancestral form. Alternatively, $P$. lodosi could be a hybrid between $P$. sanctipauli and $P$. pulcher. This is supported by the observation that there is only one known locality for $P$. lodosi, which is situated within ( $P$. sanctipauli) or at the edge ( $P$. pulcher) of the ranges of the other two species. $P$. pulcher and $P$. sanctipauli some-
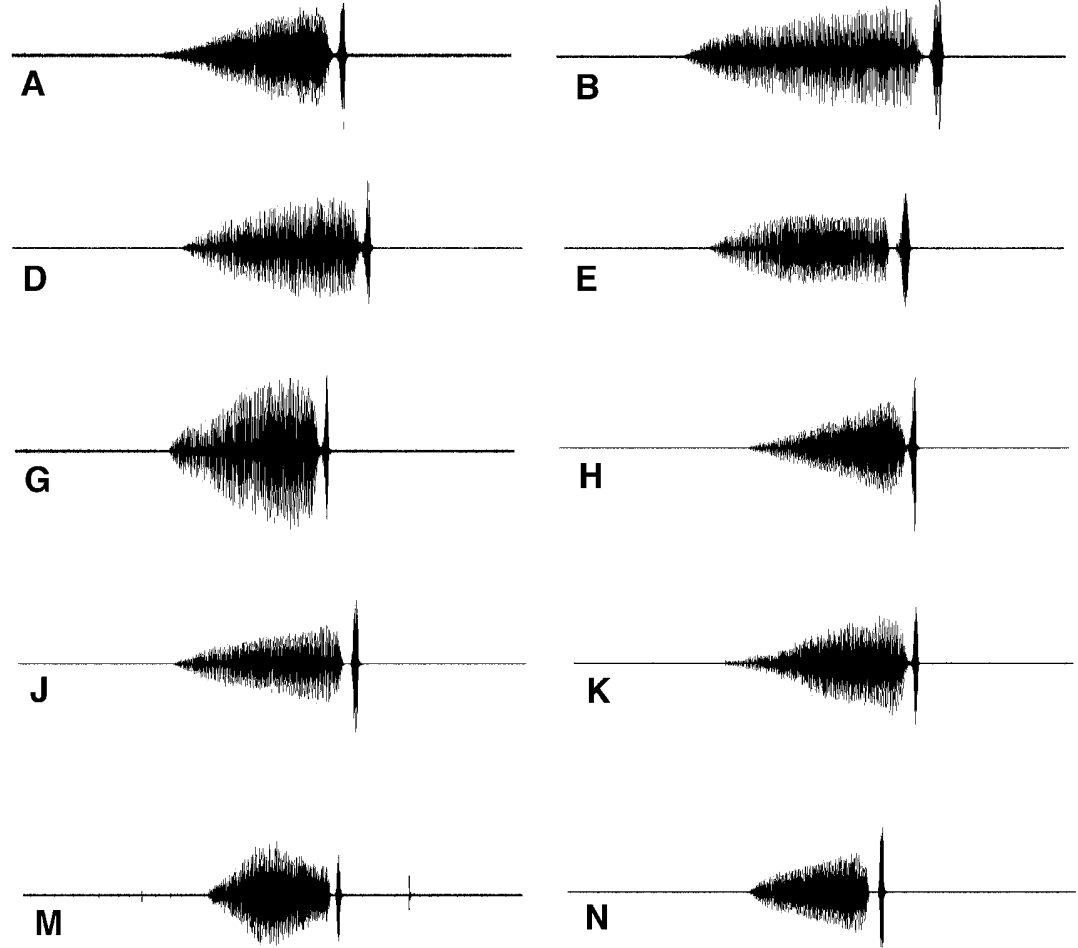
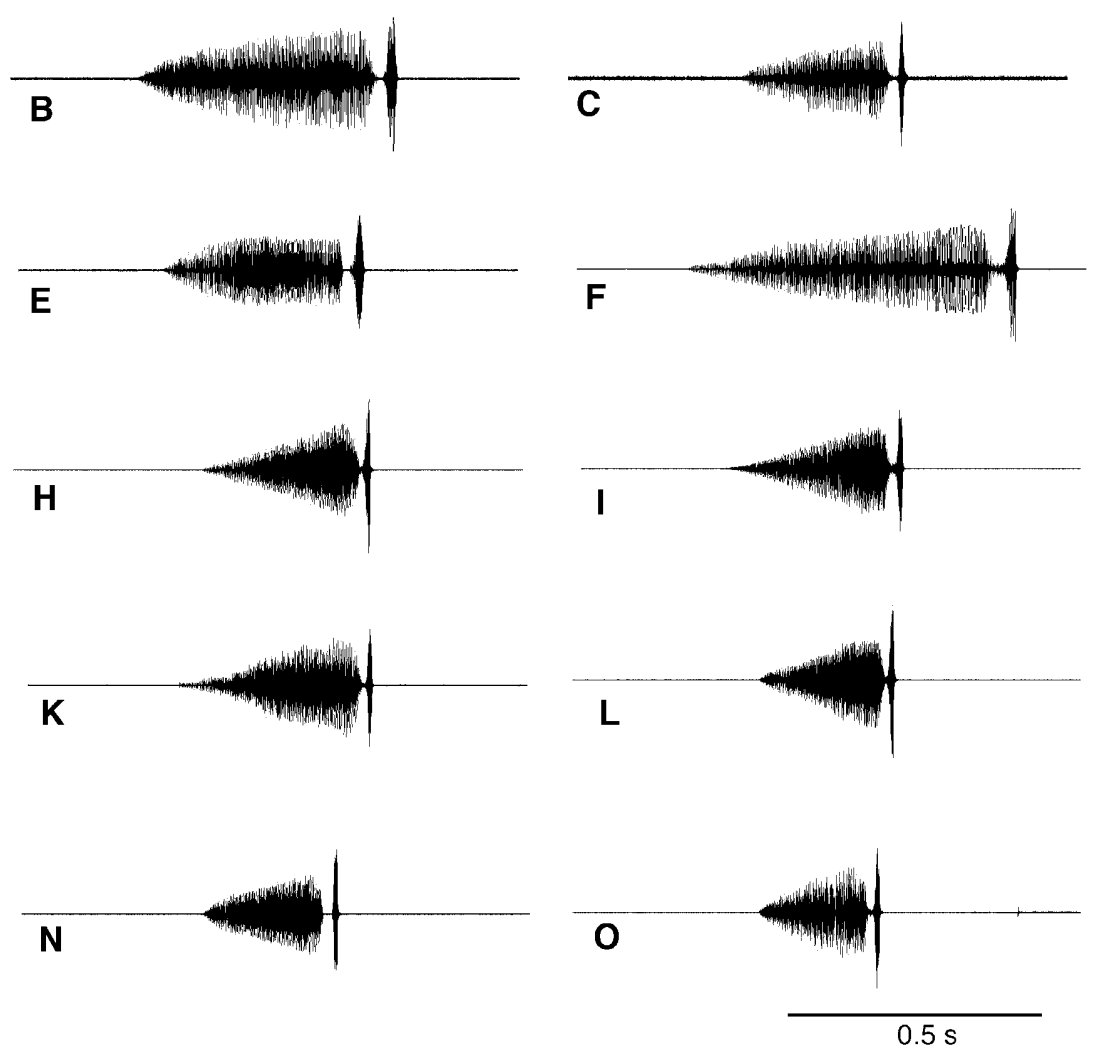

Fig. 9. Oscillograms of the calling songs of 15 males from one population of $P$. sanctipauli (Rhodos, Lindos) presented in the sequence in which they were recorded (A-F - 18 April 1983, ca. $17-18$ p.m., air temperature $18.5-16^{\circ} \mathrm{C}$; G-O - 19 April 1983 , morning, air temperature $19-21^{\circ} \mathrm{C}$ ). 


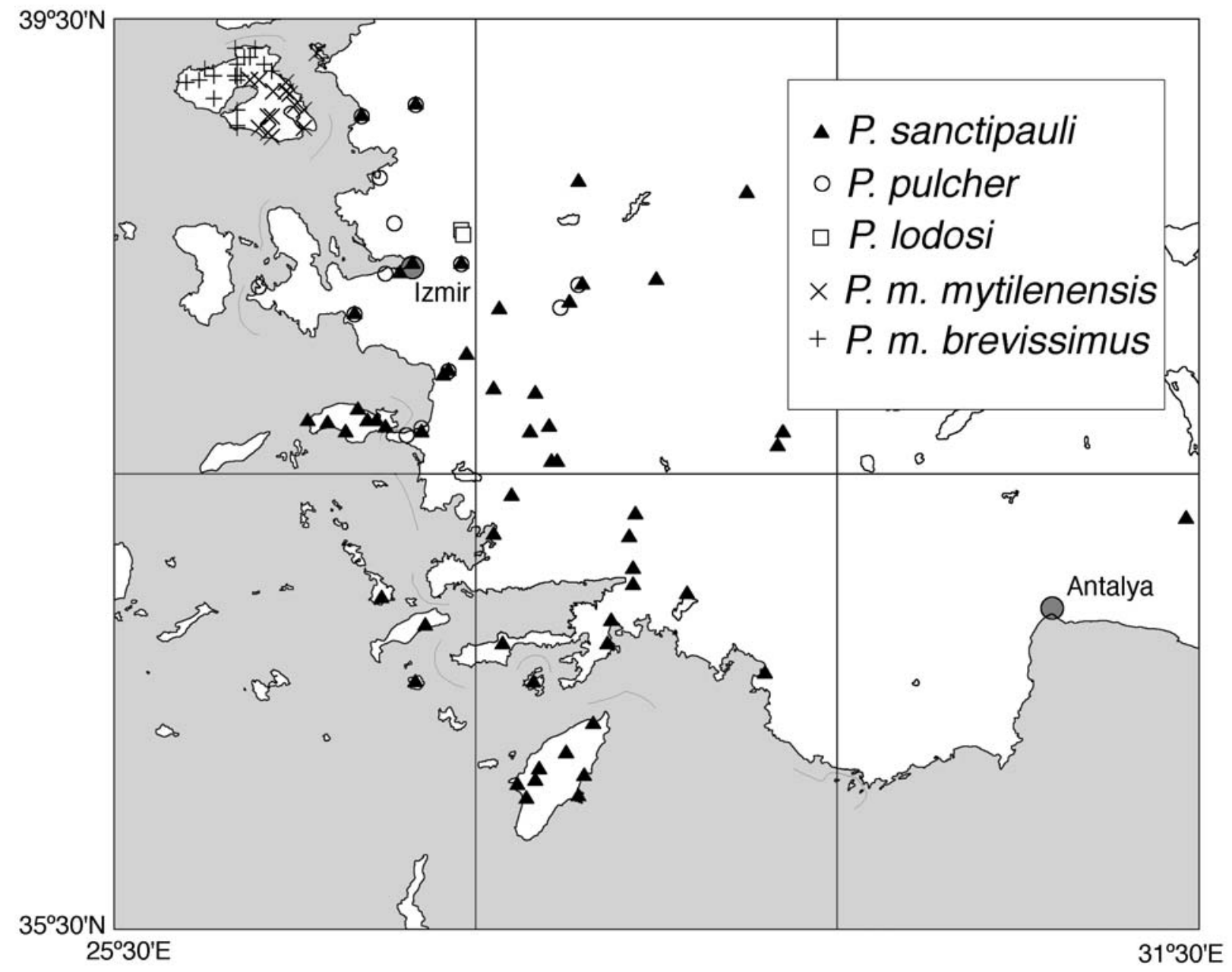

Fig. 10. Distribution map.

times occur very close together (same locality names with same or different collectors: Bergama, Selçuk, Kemalpasa, Seferihisar, Menemen, Boz Dagh). Considering their broad habitat requirements both species probably occur syntopically, but none of these localities have been examined for hybrids. At Sipil Dagh, the locality of $P$. lodosi, neither of the other two species were observed. Possibly at this locality both parent species met and the isolation barriers broke down resulting in the large hybrid population called $P$. lodosi. Hybridization occurs quite frequently when previously geographically separated forms meet. Closer examination sometimes shows that there is a complex pattern of local hybridization and syntopic co-existence of both forms. Mosaic hybrid zones with local break down of the isolation barriers between closely related species are reported in Orthoptera with increasing frequency as knowledge of these insects increases (e.g., acridid grasshoppers: Stumpner \& Helversen, 1994; Ingrisch, 1995; Vedenina \& Helversen, 2003; Bailey et al., 2004; crickets: Britch et al., 2001).

\section{Biogeography}

In the range of the genus Poecilimon, species of the $P$. sanctipauli group occur near to the south-eastern edge, an area with a high diversity of species (see Çıplak et al., 1993, 1999). They may have originated from a more widespread species, possibly a southern representative of the Balkan P. ornatus/affinis group (see Results). If this is correct the separation of the $P$. sanctipauli-group from its relatives may have occurred between the middle and late Miocene (Welter-Schultes \& Williams, 1999; Langhian to Messenian, 16.5 to 6.5 mio years ago, possibly around the Serravalian-Tortonian boundary 11 mio years ago (Dermitzakis, 1990)) when the sea flooded into the Aegean. According to preliminary DNA studies species of Poecilimon may be relatively old with divergence within groups of closely related species estimated to have occurred 8 million years ago (Lehmann, 1998). The sequence differences between $P$. lodosi and species of the $P$. ornatus/affinis group are not smaller than the ingroup differences (same gene; Pollmann \& Reinhold, unpubl.). As a first step in speciation, a northern form (today $P$. mytilenensis) may have separated from a precursor of the southern species P. sanctipauli, P. pulcher and P. lodosi, 
as indicated by their present day distribution (Fig. 10) and differences in song and morphology. The occurrence of $P$ sanctipauli on many Aegean islands as well as on the main land is not unexpected since even as recently as the pleistocene and before there were land bridges to and between the islands, and them and the mainland. However, $P$. sanctipauli is found also on the island of Nisyros, a volcanic island risen above sea level not more than 1 mio years ago and never connected with the mainland (Papanikolaou \& Lekkas, 1991). From these informations it is more surprising that $P$. mytilenensis is restricted to the islands of Lesbos and Alibey. However, the coastal mainland north of Izmir seems to be either generally poor in species (climatic reasons?) or less well studied (see distribution maps in Çıplak et al., 1993). Other details of the distribution pattern of the species, however, are more difficult to understand. Currently we cannot explain the distribution of the two subspecies of $P$. mytilenensis, which meet at a southwest-northeast borderline on the island of Lesbos (Heller et al., 2005). The distribution of $P$. pulcher is even more enigmatic (for $P$. lodosi see above). If allopatric speciation occurred (and a priori there are no reasons to invoke other theories) then it should have been isolated from $P$. sanctipauli for some time. But the ranges of $P$. pulcher and $P$. sanctipauli completely overlap. Both species live from sea level up to at least $1000 \mathrm{~m}$, so it is not easy to imagine isolation occurring during cold periods. Isolation during a very warm interglacial seems also unlikely since $P$. sanctipauli occurs on islands without high mountains where it should have become extinct during warm periods. Further studies, especially of DNA sequences, may help elucidate the age and unusual evolutionary history of the three species $P$. sanctipauli, pulcher and lodosi.

ACKNOWLEDGEMENTS. We thank M. Volleth, A Demirsoy, Y. Durmus, M. Gebhardt and A. Stumpner for their help in collecting specimens. We are grateful to T. Wasserthal for his permission to use the SEM and W. Heimler for his technical assistance (both of the Institut für Zoologie I, Universität Erlangen). Dr. Heyse, Institut für Werkstofftechnik und Werkstoffprüfung, Otto-von-Guericke-Universität Magdeburg, greatly assisted in producing another series of SEM pictures. A. Kaltenbach, Naturhistorisches Museum Wien, kindly provided information on the types of $P$. sanctipauli and P. pulcher, B. Çıplak, Fer Willemse made helpful comments on an earlier version of this paper.

\section{REFERENCES}

Bailey R.I., Thomas C.D. \& Butlin R.K. 2004: Premating barriers to gene exchange and their implications for the structure of a mosaic hybrid zone between Chorthippus brunneus and C. jacobsi (Orthoptera: Acrididae). J. Evol. Biol. 17: 108-119. Bey-Bienko G.YA. 1954: Orthoptera Vol. II, Sect. 2. BushCrickets. Subfam. Leaf Bush-Crickets (Phaneropterinae). Fauna USSR 59 (N.S.). Inst. Zool. Acad. Sci. URSS, 385 pp.

Britch S.C., CAIN M.L. \& Howard D.J. 2001: Spatio-temporal dynamics of the Allonemobius fasciatus - A. socius mosaic hybrid zone: A 14-year perspective. Mol. Ecol. 10: 627-638

BRUNNER VON Wattenwyl C. 1878: Monographie der Phaneropteriden. Brockhaus, Wien, 401 pp., 8 pls.
Brunner von Wattenwyl C. 1891: Additamenta zur Monographie der Phaneropteriden. Verh. K. K. Zool.-Bot. Ges. Wien 41: 1-196, pl. 1-2.

Chopard L. 1939: Voyage zoologique d'Henri Gadeau de Kerville en Asie-Mineure (Avril-Mai 1912). Vol. 1. Part 1. Paris, pp. 80-82.

Çiplak B., Demirsoy A. \& Bozcuk N. 1993: Distribution of Orthoptera in relation to the Anatolian diagonal. Articulata 8: $1-20$.

Çiplak B., Yalim B. \& Demirsoy A. 1999: Orthoptera fauna of Turkey. In Demirsoy A. (ed.): General Zoogeography and Zoogeography of Turkey: Animal Zoogeography. Meteksan, Ankara, pp. 748-770 (in Turkish).

Çíplak B., Yalim B., Demirsoy A. \& Sevgili H. 2002: Orthoptera fauna of Turkey. In Demirsoy A. (ed.): General Zoogeography and Zoogeography of Turkey: Animal Zoogeography. Meteksan, Ankara, pp. 681-707 (in Turkish).

DeRMITZAKIS M.D. 1990: Paleogeography, geodynamic process and event stratigraphy during the late Cenozoic of the Aegean Area. Atti Conv. Lincei 85: 263-288.

Harz K. 1969: Die Orthopteren Europas 1. Series entomologica 5. W. Junk, The Hague, $x x+749$ pp.

HARZ K. 1975: Neue Orthopterenarten und Unterarten aus der Palaearktis. Articulata 1: 5-16.

Giglio-Tos E. 1914: Escursioni zoologiche del Dr. Enrico Festa nell'Isola di Rodi. IX. Dermaptera et Orthoptera. Boll. Mus. Zool. Anat. Comp. Univ. Torino 29(680): 1-7.

Heller K.-G. 1984a: Zur Bioakustik und Phylogenie der Gattung Poecilimon (Orthoptera, Tettigoniidae, Phaneropterinae). Zool. Jb. Syst. 111: 69-117.

Heller K.-G. 1984b: Die "Isophya"-Arten von Rhodos (Orthoptera, Tettigonioidea, Phaneropteridae). Articulata 2: 74-77.

HeLLER K.-G. 1988: Bioakustik der europäischen Laubheuschrecken. Josef Margraf, Weikersheim, 358 pp.

Heller K.-G. \& Helversen D. von 1986: Acoustic communication in phaneropterid bushcrickets: species-specific delay of female stridulatory response and matching male sensory time window. Behav. Ecol. Sociobiol. 18: 189-198.

Heller K.-G. \& Lehmann A. 2004: Taxonomic revision of the European species of the Poecilimon ampliatus-group (Orthoptera: Tettigonioidea: Phaneropteridae). Mem. Soc. Entomol. Ital. 82[2003]: 403-421.

Heller K.-G. \& Reinhold K. 1992: A new bushcricket of the genus Poecilimon from the Greek islands (Orthoptera: Phaneropterinae). Tijdschr. Entomol. 135: 163-168.

Heller K.-G., Korsunovskaya O., Ragge D.R., Vedenina V., Willemse F., Zhantiev R.D. \& Frantsevich L. 1998: Checklist of European Orthoptera. Articulata 7 (Beiheft): 1-61.

Heller K.-G., Orci K.M., Grein G. \& Ingrisch S. 2004: The Isophya species of Central and West Europe (Orthoptera: Tettigonioidea: Phaneropteridae). Tijdschr. Entomol. 147: 237-258.

Heller K.-G., Willemse F. \& Sevgili H. 2005: Poecilimon mytilenensis Werner, a polytypic phaneropterid bushcricket from the Aegean islands (Orthoptera, Tettigonioidea), differing in male mating structures. J. Orthopt. Res. (in press).

ICZN (International Commission on Zoological Nomenclature) 1999: International Code of Zoological Nomenclature, 4th ed. International Trust for Zoological Nomenclature, London.

INGRISCH S. 1995: Evolution of the Chorthippus biguttulus group (Orthoptera, Acrididae) in the Alps, based on morphology and stridulation. Rev. Suisse Zool. 102: 475-535.

JACOBSON G.G. 1905: Orthoptera. In Jacobson G.G. \& Bianchi V.L. (eds): Orthopteroid and Pseudoneuropteroid Insects of Russian Empire and Adjacent Countries. Devriena, St. Petersburg, pp. 29-466. 
JANNONE G. 1936: Nuovi contributi alla conoscenza della fauna delle Isole Italiane dell'Egeo. V. Studio bio-ecologico e sistematico dell; Ortotterofauna con notiziesui Blattodei, Mantodei e Fasmoidei. Boll. Lab. Zool. Portici 29: 47-248.

Karabag T. 1958: The Orthoptera fauna of Turkey. T.C. Ankara Univ. Fen Fak. Yayinl. Istanb. 81: i-xiii, 1-198.

Karabag T., Balamir S., Gümüssuyu I. \& Tutkun E. 1971: Orthoptera fauna of Turkey (in Turkish). Bitki Koruma Bült. (Ankara) 11: 73-100.

Karabag T., GÜMüssuYu I. \& TutKun E. 1980: Orthoptera fauna of Turkey (III) (in Turkish). Bitki Koruma Bült. (Ankara) 20: $1-25$.

Lehmann A. 1998: Speciation, Acoustic Communication and Sexual Selection in Greek Bush-Crickets of the Poecilimon propinquus Group (Tettigonioidea, Phaneropteridae). $\mathrm{PhD}$ Thesis, University Erlangen-Nürnberg, 134 pp. (in German).

KIRBy W.F. 1906: A Synonymic Catalogue of Orthoptera. Vol. 2. Orthoptera Saltatoria. Part 1. (Achetidae et Phasgonuridae). British Museum, London, viii + $562 \mathrm{pp}$.

Naskrecki P. \& Otте D. 1999: An Illustrated Catalog of Orthoptera Vol. 1. Tettigonioidea (Katydids or Bush-Crickets). CD-Rom published by the Orthopterists' Society at the Academy of Natural Sciences of Philadelphia.

Önder F., Pehlivan E., Karsavuran Y., Tezcan S. \& Kismali S. 1999: Catalogue of the collection of Tettigoniidae (Orthoptera) preserved in the Prof. Dr. Niyazi Lodos Museum, Izmir, Turkey. Turk. Entomol. Derg. 23: 243-250.

Отте D. 1997: Orthoptera Species File 7. Tettigonioidea. Orthopterists' Society at the Academy of Natural Sciences of Philadelphia, Philadelphia, 373 pp.

Papanikolaou D.J. \& LekKas E.I. 1991: Geological structure and evolution of the Nisyros volcano. Bull. Geol. Soc. Greece 25: 405-419.

Ramme W. 1933: Beiträge zur Kenntnis der palaearktischen Orthopterenfauna (Tettig. et Acrid.). II. Mitt. Zool. Mus. Berlin 18: 416-434, pl. 12.

Ramme W. 1951: Zur Systematik, Faunistik und Biologie der Orthopteren von Südost-Europa und Vorderasien. Mitt. Zool. Mus. Berlin 27: 1-432, pls 1-39.

SevgiLi H. 2001: A new bushcricket species and notes on some less known species of the genus Poecilimon Fischer, 1853 from Turkey (Orthoptera, Phaneropterinae). J. Orthopt. Res. 10: $15-24$.

StumpNer A. \& Heller K.-G. 1992: Morphological differences of the auditory system in three related bushcrickets (Orthoptera: Phaneropteridae, Poecilimon). Physiol. Entomol. 17: 73-80.

StumpNer A. \& Helversen O. von 1994: Song production and song recognition of sibling species (Chorthippus dorsatus, Ch. dichrous and Ch. loratus: Orthoptera, Acrididae). Bioacoustics 6: 1-23.
TAZegül E. \& ÖNDER F. 1995: Systematical studies on the species of Tettigoniidae (Orthoptera) family in Izmir (Turkey). Ege Üniv. Fen Biliml. Inst. Derg. 5: 1-6.

ÜNAL M. 1999: Notes on Orthoptera of western Turkey, with description of a new genus and four new species. J. Orthopt. Res. 8: 243-255.

ÜnAL M. 2003: Poecilimon Fischer (Orthoptera: Tettigoniidae: Phaneropterinae) species of the Bati Karadeniz Region of Turkey, N.W. Anatolia. Trans. Am. Entomol. Soc. 129: 361-383.

Vedenina V.Y. \& Helversen O.V. 2003: Complex courtship in a bimodal grasshopper hybrid zone. Behav. Ecol. Sociobiol. 54: $44-54$.

Welter-Schultes F.W. \& Williams M.R. 1999: History, island area and habitat availability determine land snail species richness of Aegean islands. J. Biogeograph. 26: 239-249.

Werner F. 1901: Die Dermapteren- und Orthopterenfauna Kleinasiens. S.B. Akad. Wiss. Wien, Math.-Nat. Kl., Abt. 1 110: 259-306, pls 1-2.

WERNER F. 1932: Neue griechische Orthopteren aus dem Gebiet des Ägäischen Meeres, I. Anz. Akad. Wiss. Wien 27: 294-296.

WERNER F. 1933: Ergebnisse einer zoologischen Studien- und Sammelreise nach den Inseln des Ägäischen Meeres II. Orthopteren. S.B. Akad. Wiss. Wien, Math.-Nat. Kl., Abt. 1 142: 185-204.

WERNER F. 1936: Ergebnisse einer zoologischen Forschungsreise nach dem Dodekanes, unternommen von Kustos Dr. Otto Wettstein. I. Orthopteren aus dem Dodekanes. S.B. Akad. Wiss. Wien, Math.-Nat. Kl., Abt. 1 145: 9-16.

WiLLEMSE F. 1982: A survey of the Greek species of Poecilimon Fischer (Orthoptera, Ensifera, Phaneropterinae). Tijdschr. Entomol. 125: 155-203.

Willemse F. 1984: Catalogue of the Orthoptera of Greece. Vol. 1. Fauna Graeciae, Hellenic Zoological Society, Athens, xii + 275 pp.

WILLEMSE F. 1985: A key to the Orthoptera Species of Greece. Vol. 2. Fauna Graeciae, Hellenic Zoological Society, Athens $288 \mathrm{pp}$.

Willemse F. \& Heller K.-G. 1992: Notes on systematics of Greek species of Poecilimon Fischer, 1853 (Orthoptera: Phaneropterinae). Tijdschr. Entomol. 135: 299-315.

\section{DATABANKS}

SysTax (a Database System for Systematics and Taxonomy): http://www.biologie.uni-ulm.de/systax/daten/index.html

DORSA (Deutsche Orthopteren Sammlungen - German Orthoptera collections: http://www.dorsa.de/

OSF2 Orthoptera Species File online version 2.1: http://140.247.119.145/Orthoptera* former/other versions: http://viceroy.eeb.uconn.edu/Orthoptera (20/11/1998) and http://www.tettigonia.com (13/11/2003)

Received June 17, 2004; revised and accepted November 30, 2004 